\title{
Rhubarb-Aconite Decoction (RAD) Drug-Containing Serum Alleviated Endotoxin-Induced Oxidative Stress Injury and Inflammatory Response in Caco-2 Cells In Vitro
}

\author{
Xiao-hong Du, ${ }^{1}$ Qing-jun Chen, ${ }^{2}$ Jian-bo Song, ${ }^{3}$ Yan Xie, ${ }^{4}$ Yan Zhi, ${ }^{5}$ Ru-ru Sun, ${ }^{2}$ \\ Guo-hui Liu $\mathbb{D}^{6},{ }^{6}$ and Xin Kang $\mathbb{C}^{2}$ \\ ${ }^{1}$ Department of Hepatobiliary Pancreatic Surgery, First Hospital Bethune of Jilin University, Changchun 130021, China \\ ${ }^{2}$ Department of Emergency Medicine, Beijing Hepingli Hospital, Beijing 100013, China \\ ${ }^{3}$ Department of Emergency Medicine, Affiliated Zhongshan Hospital, Dalian University, Dalian 116001, China \\ ${ }^{4}$ Organ Transplant Center, Tianjin First Central Hospital, Tianjin 300000, China \\ ${ }^{5}$ Department of Burns, The Second Affiliated Hospital of Kunming Medical University, Kunming 650101, China \\ ${ }^{6}$ Department of Emergency Medicine, The First Hospital of Jilin University, Changchun 130012, China
}

Correspondence should be addressed to Guo-hui Liu; ghliu@jlu.edu.cn and Xin Kang; 254635652@qq.com

Received 29 February 2020; Accepted 25 May 2020; Published 4 July 2020

Academic Editor: Andresa A. Berretta

Copyright (C) 2020 Xiao-hong Du et al. This is an open access article distributed under the Creative Commons Attribution License, which permits unrestricted use, distribution, and reproduction in any medium, provided the original work is properly cited.

\begin{abstract}
Rhubarb-Aconite Decoction (RAD), a famous Chinese medicine prescription, has been widely used for treating intestinal injury. However, the effect of RAD on intestinal epithelial cells is unclear. The aim of this study was to investigate the effects of RAD drugcontaining serum on the oxidative stress injury and inflammatory response induced by endotoxin (ET) in Caco-2 cells in vitro. Lipid peroxide malondialdehyde (MDA), lactate dehydrogenase (LDH), caspase-11, tumor necrosis factor- $\alpha$ (TNF- $\alpha$ ), interleukin3(IL-3), and cytokeratin (CK)18, adenosine triphosphate (ATP) activity, and intracellular free calcium ion levels were measured. The results showed that ET triggered the activation of caspase- 11 and the massive release of TNF- $\alpha$, increased the inhibitory rate of cell growth, MDA, and LDH expressions in Caco-2 cells. Moreover, RAD drug-containing serum could inhibit caspase-11 activation, decrease the release of TNF- $\alpha$ and IL-3, reduce intracellular free calcium ion, and enhance CK 18 expression and ATP activity. These novel findings demonstrated that ET-induced oxidative stress injury and inflammatory response of Caco-2 cells were improved by RAD drug-containing serum, indicating that RAD may be a good choice for the treatment of intestinal injury.
\end{abstract}

\section{Introduction}

The intestine is the core organ of posttraumatic stress and the initiating organ of multiple organ dysfunction in the development of severe complications under critically stressful events [1-3], including trauma [4], burns [5], and brain injury $[6,7]$. These stressful events may initiate a cascade of intestinal events, including the destruction of the intestinal mucosa, barrier dysfunction, translocation of intestinal bacteria, and endotoxin (ET), a major component of the outer membrane of Gram-negative bacteria, which may cause systemic inflammatory response syndrome (SIRS) and multiple organ dysfunction syndrome (MODS) [8]. The intestinal epithelial is known as the most critical barrier for protection against harmful antigens and pathogens [9]. Oxidative stress injury and inflammatory response have been implicated in the dysfunction of the intestine barrier. It is necessary to reduce oxidative stress injury and inhibit the inflammatory response to protect the normal structure and function of intestinal epithelial cells. At present, people often use chemical methods to synthesize antioxidants, but animal experiments show that they have certain toxicity and carcinogenic effects [10-12]. Thus, natural antioxidants are urgently needed, and Traditional Chinese medicine (TCM) is deemed promising to avoid the oxidative injury of intestine. 
Rhubarb-Aconite Decoction (RAD), a famous Chinese medicine prescription, was originally described in Chinese Medical Classics-Jin Kui Yao Lue. RAD consists of Radix et Rhizoma Rhei, Radix Aconiti Lateralis Praeparata, and Radix et Rhizoma Asari, which has been widely used for intestinal obstruction, chronic diarrhea, and intestinal injury [13-15]. The study showed that RAD reduced serum ET level, stimulated intestinal peristalsis, and protected intestinal mucosal barrier function in patients with severe acute pancreatitis $[16,17]$. However, the effect of RAD on the role of intestinal epithelial cells is unclear. The serum pharmacological method of TCM, a semidetached residential in vivo experiment method, has obvious advantages in the study of the pharmacological effects of TCM $[18,19]$. Serum pharmacological methods may contribute to the study of the effects of RAD on intestinal epithelial cells in vitro. So, the aim of this study was to investigate the effects of RAD drugcontaining serum on the oxidative stress injury and inflammatory response induced by ET in human Caco- 2 cells in vitro.

\section{Materials and Methods}

2.1. Materials and Reagents. Human colon Caco-2 cells were obtained from the American Type Culture Collection (Rockville, MD, US). Anti-CK 18 antibody was supplied by Abcam plc (Cambridge, UK). Dulbecco's modified eagle medium (DMEM), fetal bovine serum (FBS), L-glutamine, nonessential amino acids, penicillin, and streptomycin were purchased from Life Technologies (Carlsbad, CA, USA). ET derived from Escherichia coli 0127:B8 and Thiazolyl Blue tetrazolium bromide (MTT) were all from Sigma (St. Louis, MO, the United States). Malondialdehyde (MDA), Adenosine triphosphate (ATP), and lactate dehydrogenase (LDH) assay kits were obtained from Nanjing Jiancheng Bioengineering Institute (Nanjing, Jiangsu, China). Enzyme-linked immunosorbent assay (ELISA) kits and Western blot kits of caspase-11, tumor necrosis factor $\alpha$ (TNF- $\alpha$ ), and interleukin 3 (IL-3) were from Elabscience Biotechnology Co.Ltd (Elabscience, Wuhan, China) and Biovision Co.Ltd (San Francisco, CA, the United States). BCA Protein Assay Kit was obtained from KeyGen Biotech. Co. Ltd (Nanjing, Jiangsu, China). Ponceau S was purchased from Puhe Biological Medicine Technology Co., Ltd (Wuxi, Jiangsu, China). Fluo-3-am was obtained from Life Technologies Corporation (Paisley, UK). Tween-20 was obtained from AMRESCO, Ltd. (Solon, OH, the United States) and OsO4 from Amresco, Ohio, the United States. All other chemicals and reagents used were of analytical grade.

\subsection{Preparation of RAD Drug-Containing Serum}

2.2.1. Animals. Six SD rats (aged eight weeks, weighted $250 \pm 20 \mathrm{~g}$ ) were provided by the Dalian Medical University Experimental Animal Center (Dalian, Liaoning, China), which were housed in the institutional animal facility with standard animal room conditions $\left(25^{\circ} \mathrm{C}, 12\right.$-hour light-dark cycles, $\leq 3$ animals in a cage). All animals used in this study were cared for in compliance with the Guide for the Care and Use of Laboratory Animals. All experimental procedures using live animals were approved by the Animal Research Ethics Committee of Affiliated Zhongshan Hospital of Dalian University. Anesthetic drugs and all other necessary measures were used to reduce animal suffering during experimental procedures.

2.2.2. Serum Preparation. RAD is composed of 3 species of herbal plants (rhubarb, aconite, and Asarum), each dried crude drug of which were purchased from Tong Ren Tang Group Co., Ltd (Beijing, China). Rhubarb (9g), aconite (9g), and Asarum ( $3 \mathrm{~g}$ ) were mixed in the ration of $3: 3: 1(\mathrm{w} / \mathrm{w})$. First, aconite was soaked in water $(1: 25)$ for $30 \mathrm{mins}$, followed by extraction in boiling water $\left(100^{\circ} \mathrm{C}\right)$ for $1 \mathrm{~h}$. Then, rhubarb was added and boiled for $10 \mathrm{~min}$. Finally, Asarum was added and boiled for 5 mins, and RAD was obtained.

RAD drug-containing serum was prepared by serum pharmacological method [19, 20]. SD rats were divided into two groups for preparing RAD drug-containing serum and blank serum, respectively. One group of rats $(n=3)$ were given RAD through gavage at the doses of $20 \mathrm{~g} /(\mathrm{kg} \cdot \mathrm{d})$ for three days. After $2 \mathrm{~h}$ of the last treatment, abdominal aortic blood was collected under aseptic condition and then centrifuged at 3,000 r/min for 15 mins to obtain RAD drug-containing serum (RAD-CS). The other group of rats $(n=3)$ were given normal saline, and then obtained blank serum (BS). All serum was filtered through $0.22 \mu \mathrm{m}$ filter membrane and inactivated by water bath at $56^{\circ} \mathrm{C}$ for 30 mins, and then stored at $-20^{\circ} \mathrm{C}$ until being used for the pharmacological study.

2.3. Cell Culture, Passage, and Grouping. Caco-2 cells were cultured in DMEM medium supplemented with $10 \%$ heatinactivated FBS, 4.0 mM L-glutamine, 1\% nonessential amino acids, $100 \mathrm{U} / \mathrm{ml}$ penicillin, and $100 \mu \mathrm{g} / \mathrm{mL}$ streptomycin, and maintained in an incubator with $5 \% \mathrm{CO}_{2}$ humidified at $37^{\circ} \mathrm{C}$. When the degree of cell confluence reached $80 \%$, cell passage was carried out. The medium was changed every other day until the cells were fully differentiated. Caco2 cells between the 50th to 60th passages were seeded on the apical compartment of Transwell ${ }^{\circledR}$ plates at a density of $1 \times 10^{4}$ cells/well and were inoculated in an incubator with $5 \% \mathrm{CO}_{2}$ at $37^{\circ} \mathrm{C}$ for $24 \mathrm{~h}$. Then, the experiments were conducted with 4 groups: CK group (only Caco- 2 cells), Low-dose ET group (L-ET), Medium-dose ET group (MET), and high-dose ET group (H-ET). CK group cells were not stimulated with ET. The Caco-2 cells in the L-ET group, M-ET group, and H-ET group were treated with 0.1, 1.0, and $10.0 \mathrm{EIU} / \mathrm{ml}$ of ET, respectively. Cells of each group were transferred to the Petri plate and cultured together with ET for 48 hours, and then inhibitory rate, MDA, LDH, and TNF- $\alpha$ of cells treated with ET were detected according to the kit instructions. In addition, mitochondrial structure was observed under transmission electron microscope (TEM). The results of these indicators are shown in Figure 1, which proved that $10.0 \mathrm{EIU} / \mathrm{ml}$ of ET significantly induced inflammatory response and oxidative stress injury of Caco2 cells, and the cells stimulated with $10.0 \mathrm{EIU} / \mathrm{ml}$ of ET were used in subsequent experiments. 


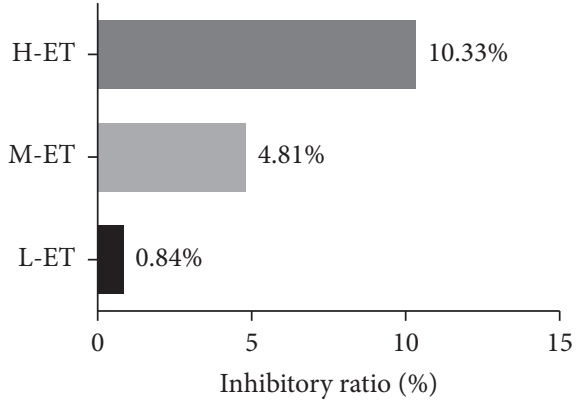

(a)

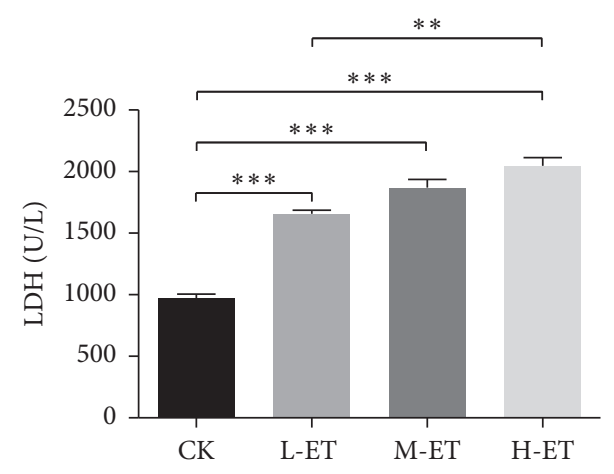

(c)

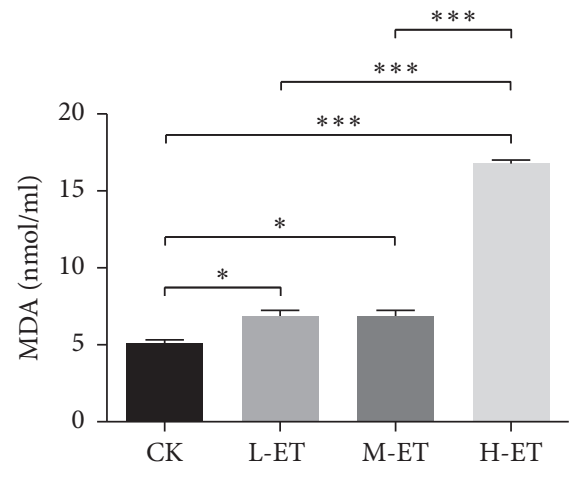

(b)

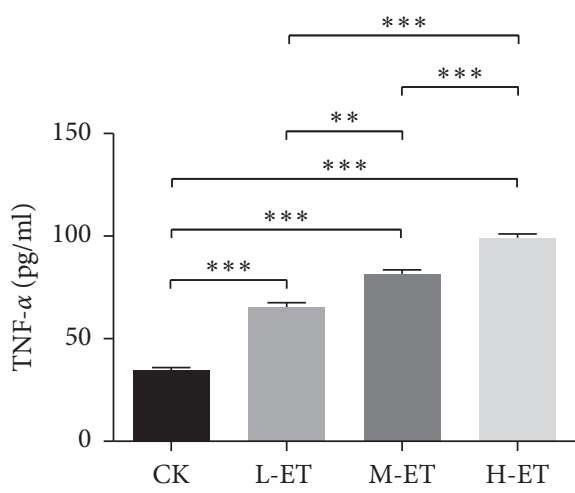

(d)

FIGURE 1: Effects of different doses of endotoxin (ET) on the inhibitory ratio of cell growth, malondialdehyde (MDA), Lactate dehydrogenase (LDH), and tumor necrosis factor- $\alpha$ (TNF- $\alpha$ ) levels of Caco-2 cells. CK group: Caco- 2 cells, L-ET group: Caco- 2 cells stimulated with Low-dose ET $(0.1 \mathrm{EIU} / \mathrm{ml}), \mathrm{M}-\mathrm{ET}$ group: Caco-2 cells stimulated with medium-dose ET (1.0 EIU/ml), H-ET group: Caco-2 cells stimulated high-dose ET (10.0 EIU/ml). Graphs show mean \pm SD. Based on one-way ANOVA statistical analysis. ${ }^{*} P<0.05,{ }^{* *} P<0.01$, ${ }^{* * *} P<0.001$.

\subsection{The Effect of RAD Containing Serum on Caco-2 Cells Stimulated by ET}

2.4.1. Cells Treatment. To investigate the effect of RAD containing serum (RAD-CS) and blank serum (BS) on 10.0 $\mathrm{EIU} / \mathrm{ml}$ ET-induced inflammatory and oxidative stress injury of Caco- 2 cells, we took Caco- 2 cells for the following experiment. These cells were divided into four groups: CK, $\mathrm{ET}, \mathrm{ET}+\mathrm{BS}$, and ET + RAD-CS groups. Caco- 2 cells in the ET group were stimulated with $10 \mathrm{EIU} / \mathrm{ml}$ of ET. ET + BS group cells were stimulated with $10 \mathrm{EIU} / \mathrm{ml}$ of ET and then cocultured with BS. ET + RAD-CS group cells were stimulated by $10 \mathrm{EIU} / \mathrm{ml}$ of ET and then cocultured with RAD-CS. The CK group cells did not have any treatment. These cells were cultured with DMEM that changed once a day, until the growth of cells filled with dish. Then, cell inhibitory ratio, antioxidant activity, inflammatory cytokines, and mitochondrial morphology changes were measured.

2.4.2. MTT Evaluated Inhibitory Ratio of Cells. The inhibitory ratio of Caco-2 cells was evaluated by MTT Cell Proliferation and Cytotoxicity Assay Kit. The concentration of Caco- 2 cells was adjusted by $1 \times 10^{5}$ cells $/ \mathrm{ml}$, then seeded and cultivated in 96 well culture plate (100ul each hole) at $37^{\circ} \mathrm{C}$ in an incubator with $5 \% \mathrm{CO}_{2}$ for $24 \mathrm{~h}$ for detecting cell inhibitory ratio by MTT. The optical density (OD) value was measured by a microplate reader (Multiskan MK3, Thermo, United State) at $492 \mathrm{~nm}$ wavelength. Inhibitory ratio $(\%)=(1-$ average OD treatment group/average OD CK group) $\times 100 \%$.

2.4.3. Mitochondrial Morphology Observation by Transmission Electron Microscopy (TEM). Caco-2 cells in each group were fixed with $0.25 \%$ glutaraldehyde (Sigma-Aldrich, MO, the United States) in $0.1 \mathrm{M}$ cacodylate buffer, $\mathrm{pH} 7.4$, for $4 \mathrm{~h}$, followed by postfixation in $2 \% \mathrm{OsO}_{4}$ in $0.1 \mathrm{M}$ cacodylate buffer for $1 \mathrm{~h}$. These cells were washed in distilled water, dehydrated in a graded series of ethanol, and then substituted with acetone, dried by sublimation in Peldri II (Plano GmbH, Marburg, Germany), sputter-coated with gold, and at last observed by TEM (HT7700, Hitachi, Japan).

2.4.4. Measurement of Caspase-11, TNF- $\alpha$, and IL-3 by ELISA and Western Blot. Caspase-11, a specific sensor for intracellular ET recognition, mediates the noncanonical 
inflammatory pathway, which was detected by ELISA. Caco2 cells concentration was adjusted to $1 \times 10^{5}$ cells $/ \mathrm{ml}$ and inoculated in a 96-well culture plate, $100 \mu \mathrm{l}$ per well, cultured in an incubator with $5 \% \mathrm{CO}_{2}$ at $37^{\circ} \mathrm{C}$ for $24 \mathrm{~h}$. Then, the supernatant was collected after $24 \mathrm{~h}$, and caspase-11 concentration was measured at $450 \mathrm{~nm}$ with the microplate reader. In addition, TNF- $\alpha$ and IL-3 expressions were also measured by ELISA kits.

Western blot was used for semiquantitative determination of expression of caspase-11, TNF- $\alpha$, and IL-3. Total proteins were extracted from Caco- 2 cells and lysates were separated by Sodium Dodecyl Sulfonate-Polyacrylamide Gel Electrophoresis (SDS-PAGE). The proteins were then transferred to polyvinylidene difluoride (PVDF) membrane (Millipore, ON) for determination of specific protein expression using a Bio-Rad Imaging System (Bio-Rad Biosciences, Hercules, CA, United States). Relative expression was normalized to $\beta$-actin.

2.4.5. LDH Measurement by Spectrophotometer. The antioxidant activity of RAD drug-containing serum on the Caco- 2 cells was evaluated by detecting LDH expression by the spectrophotometer. Caco- 2 cells concentration was adjusted to $1 \times 10^{5}$ cells $/ \mathrm{ml}$. It was vaccinated in 24 hole culture plate, $1 \mathrm{ml}$ per hole, in a $5 \% \mathrm{CO}_{2}$ incubator at $37^{\circ} \mathrm{C}$ for $24 \mathrm{~h}$. The cell supernatant was incubated for $24 \mathrm{~h}$. The cell supernatants were detected according to $\mathrm{LDH}$ kit instructions and detected at $450 \mathrm{~nm}$.

2.4.6. Expression of CK18 by Confocal Microscope. CK 18 is a characteristic marker of intestinal epithelial cells. Caco2 cells were adjusted to $1 \times 10^{5}$ cells $/ \mathrm{ml}$, inoculated in a 24 well culture plate, $1 \mathrm{ml}$ per well, incubated in a $5 \% \mathrm{CO}_{2}$ incubator at $37^{\circ} \mathrm{C}$ for $24 \mathrm{~h}$. The supernatant was aspirated, and cells were fixed with $4 \%$ paraformaldehyde for 10 mins. Endogenous peroxidase was inactivated. The cells were incubated with $3 \% \mathrm{H}_{2} \mathrm{O}_{2} / \mathrm{PBS}$ for 10 mins at room temperature, rinsed with PBS for 5 mins, and repeated 3 times. The slice was immersed in an antigen retrieval solution (trisodium citrate buffer) at $96^{\circ} \mathrm{C}$ for 5 mins, washed with $2 \mathrm{ml}$ PBS by a $1 \mathrm{ml}$ pipette, repeated 3 times ( 5 mins each time), and then closed by $10 \%$ FBS at room temperature for 15 mins. CK18 antibody was added and incubated at $4{ }^{\circ} \mathrm{C}$ overnight. The slice was washed with $2 \mathrm{ml} \mathrm{PBS}$ by a $1 \mathrm{ml}$ pipette, repeated 3 times, for 5 mins each time. Secondary antibody IgG-CY3 was added to the section and incubated at $37^{\circ} \mathrm{C}$ for 30 mins. The slice was rinsed with PBS 3 times, 5 mins per time. Cells were stained with Hoechst for 30 mins. The expression of CK18 was observed under confocal microscopy.

2.4.7. Detection of Mitochondrial ATP Concentration and Intracellular Calcium Ion by Flow Cytometry. In order to further study the effect of RAD drug-containing serum on Caco- 2 cells, the changes of mitochondria ATP concentration and intracellular free calcium ion were measured by flow cytometry. After the cells in the 24-well plate were fixed with $4 \%$ paraformaldehyde, the Fluo-3-am probe was incubated for 30 mins, the flow detection was performed, and the expression of fluorescence value was measured by flow cytometry.

2.5. Statistical Analysis. The data were expressed as mean\pm standard deviation (SD). The statistical analyses were performed with GraphPad Prism 6.0 software (San Diego, California, United States). Differences among multiple groups were assessed by one-way ANOVA. A value of $P<0.05$ was considered statistically significant.

\section{Results}

3.1. ET Inhibited Growth of Caco-2 cells. In order to determine the effect of ET on Caco-2 Cells growth, we tested the inhibitory ratio of cells by MTT. As shown in Figure 1(a), the inhibitory ratio of Caco- 2 cell stimulated with $0.1,1.0$, and $10.0 \mathrm{EIU} / \mathrm{mL}$ of ET were $0.84 \%, 4.81 \%$, and $10.33 \%$, respectively, which indicated that $10.0 \mathrm{EIU} / \mathrm{mL}$ of ET exerted maximum inhibitory ratio of Caco- 2 cell growth.

3.2. ET Caused Oxidative Stress Injury and Release of Inflammatory Cytokines of Caco-2 Cells. To evaluate the oxidative stress injury of Caco- 2 cells, MDA and LDH levels were measured. As shown in Figures 1(b) and 1(c), MDA and LDH levels in L-ET, M-ET, and H-ET groups were all higher than that in the $\mathrm{CK}$ group $(P<0.05)$, and their concentration reached the highest level after Caco-2 Cells were stimulated with $10.0 \mathrm{EIU} / \mathrm{mL}$ of ET, which indicate that ET caused obvious oxidative stress injury of Caco- 2 cells. TNF- $\alpha$, a proinflammatory factor, could promote the recruitment and activation of other inflammatory mediators. As shown in Figure $1(\mathrm{~d}), \mathrm{TNF}-\alpha$ in the H-ET groups was higher than that in the CK, L-ET, or M-ET groups, respectively $(P<0.001)$.

3.3. ET Caused Mitochondrial Injury of Caco-2Cells. Caco- 2 cells' mitochondria in the CK group were intact and its crista is distributed evenly (Figure 2(a)). Mitochondrial morphology of cells in the L-ET group and M-ET group were destroyed in different degrees (Figures 2(b) and 2(c)). In the $\mathrm{H}$-ET group, the mitochondrial structure of Caco- 2 cells was significantly damaged, membranes and crista severely dissolved, and their injury degree was the most serious compared with the other three groups (Figure 2(d)).

3.4. RAD Containing Serum Decreased the Expression of Caspase-11, TNF- $\alpha$, and IL-3 Protein Induced by ET. Caspase-11 plays a key role in recognition of intracellular ET. As depicted in Figure 3(a), ET dramatically increased the secretion of caspase-11 in Caco-2 cells compared with CK group, and RAD drug-containing serum inhibited significantly the activation of caspase- 11 induced by ET compared with blank serum according to ELISA results. In addition, Western blot results also demonstrated that the expression of caspase-11 protein in the ET group was stronger than that 


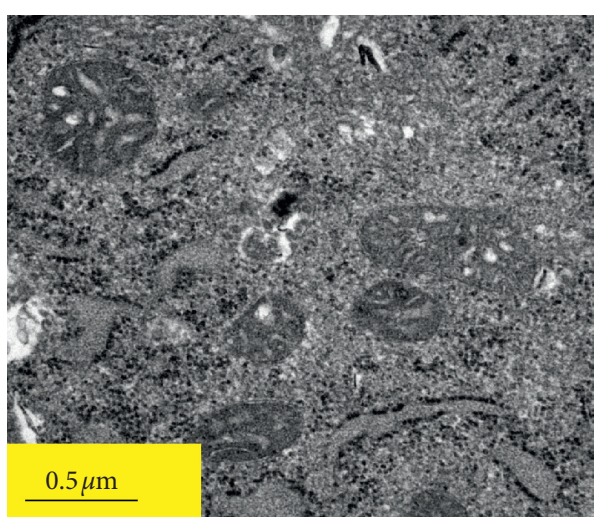

(a)

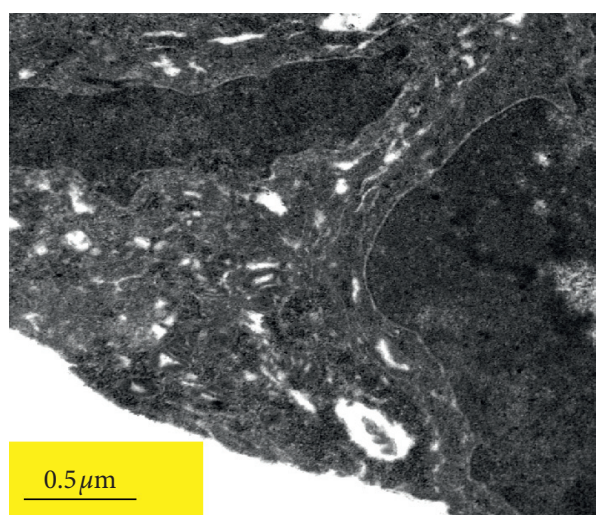

(c)

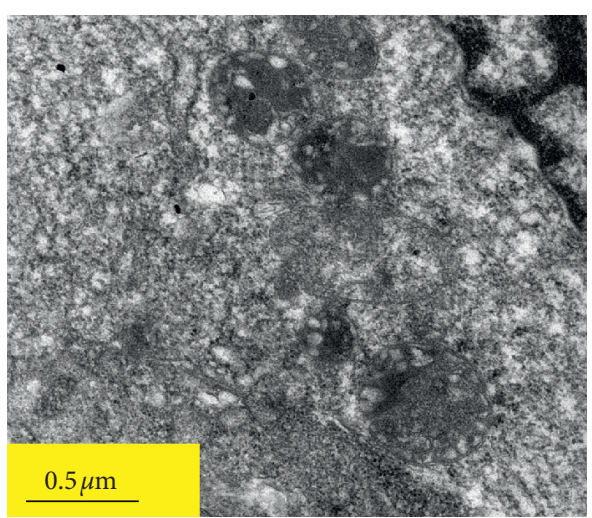

(b)

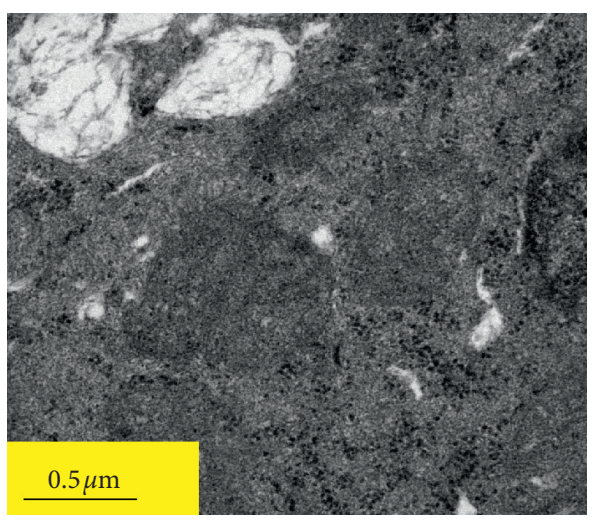

(d)

FIGURE 2: Mitochondrial changes of Caco-2 cells stimulated with different doses of endotoxin (ET) under transmission electron microscopy (TEM). (a) CK group: only Caco-2 cell, (b) L-ET group: Caco-2 cells stimulated with Low-dose ET (0.1 EIU/ml), (c) M-ET group: Caco2 cells stimulated with medium-dose ET(1.0 EIU/ml), (d) H-ET group: Caco- 2 cells stimulated high-dose ET (10.0 EIU/ml). Scale: $0.5 \mu \mathrm{m}$. Magnifications: $\times 5000$.

of the CK group, but there was no significant difference between the $\mathrm{ET}+\mathrm{BS}$ group and the ET + RAD-CS group (Figure 3(b)).

From Western blot results, the expressions of TNF- $\alpha$ and IL-3 protein in the ET group were stronger than that of CK group, and RAD containing serum reduced significantly the expression of TNF- $\alpha$ and IL-3 protein compared with the ET group and the ET+BS group (Figure 3(b)). Moreover, ELISA results also showed that RAD containing serum inhibited significantly ET-induced secretion of TNF- $\alpha$ and IL-3 (Figures 3(c) and 3(d)).

3.5. RAD Containing Serum Decreased LDH Levels. LDH levels were used to evaluate the oxidative stress injury of Caco- 2 cells. As depicted in Figure 4, ET significantly increased LDH level compared with CK group $(P<0.001)$, while LDH levels in ET + RAD-DS group were higher than those in ET + BS group and ET group, which suggested that RAD containing serum could decrease LDH levels induced by ET (Figure 4).

3.6. RAD Containing Serum Enhanced CK18 Expression of Caco-2 Cells. CK18, a marker factor of epithelial cells, was analyzed by immunofluorescence. As shown in Figure 5(a), CK18 expressions of Caco-2 cells in the CK group were the strongest among the 4 groups, and which were significantly weakened in the ET group compared with the GK group. Further, we also found that the expression of CK18 of Caco2 cells was enhanced after the addition of RAD drug-containing serum. These immunofluorescence results suggested that RAD containing serum alleviated Caco- 2 cells injury was induced by ET.

3.7. Effect of RAD Containing Serum on ATP and Intracellular Free Calcium Ion Levels. As shown in Figure 5(b), ATP levels in the ET group were significantly lower than that of the CK group $(P<0.001)$, ATP levels in the $\mathrm{ET}+\mathrm{BS}$ group, and the $\mathrm{ET}+\mathrm{RAD}-\mathrm{CS}$ group were higher than that of the ET group $(P<0.001)$. As shown in Figure 5(c), ET increased significantly the intracellular free calcium ion level, which was reversed by RAD containing serum, while blank serum was not $(P<0.05)$.

\section{Discussion}

The major findings of our study can be summarized as follows. (1) ET triggered the activation of caspase-11 and the 


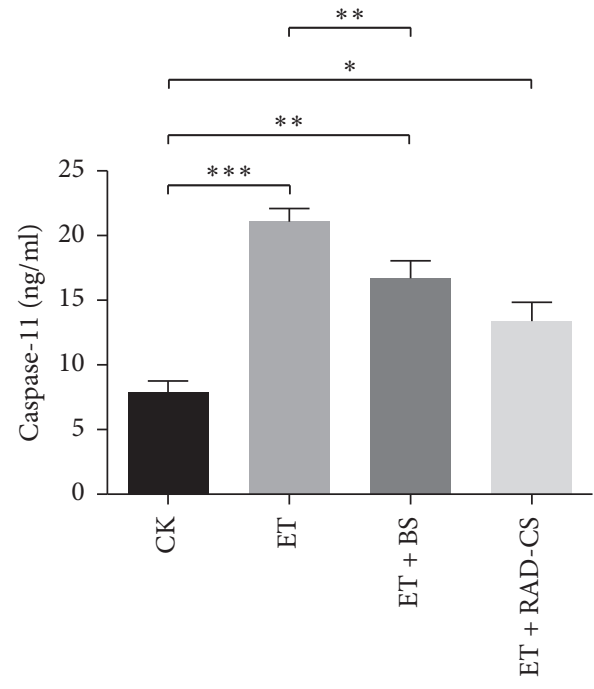

(a)

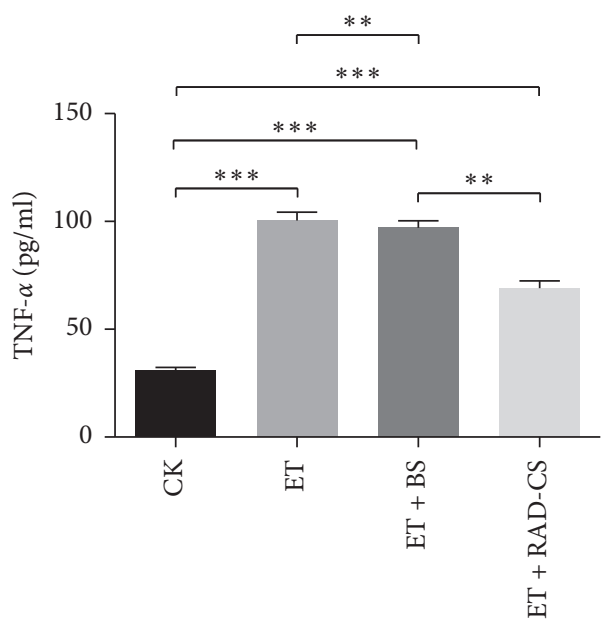

(c)

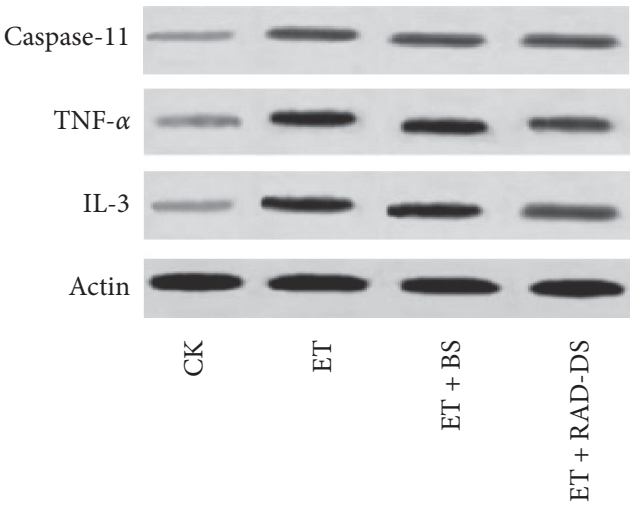

(b)

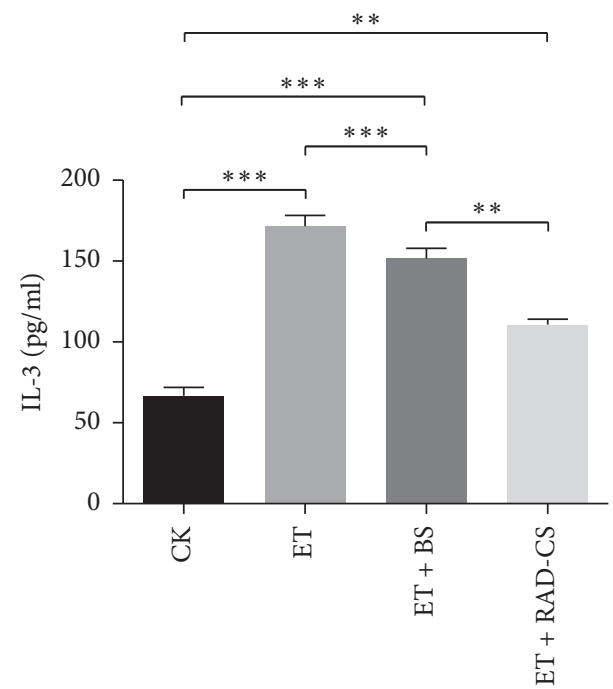

(d)

FigURE 3: Effects of RAD containing serum on caspase-11, tumor necrosis factor- $\alpha$ (TNF- $\alpha$ ), and interleukin-3 (IL-3) in Caco-2 cells induced by endotoxin (ET) by ELISA and Western blot. BS: blank serum. RAD-DS: RAD drug-containing serum. ET group: Caco- 2 cells were stimulated with $10 \mathrm{EIU} / \mathrm{ml}$ of ET, ET + BS group: cells were stimulated with $10 \mathrm{EIU} / \mathrm{ml}$ of ET and then cocultured with BS, ET + RADCS group: cells were stimulated with $10 \mathrm{EIU} / \mathrm{ml}$ of ET and then cocultured with RAD-CS, CK group: control group. (a, c, d) based on ELISA analysis, (b) based on Western blot analysis. Graphs show mean \pm SD. Based on one-way ANOVA statistical analysis. ${ }^{*} P<0.05,{ }^{* *} P<0.01$, and ${ }^{* * *} P<0.001$.

massive release of TNF- $\alpha$ and IL-3 increased the MDA and LDH levels, which could successfully induce an inflammatory response and oxidative stress injury of Caco-2 cells. (2) RAD drug-containing serum could alleviate ET-induced inflammatory response and oxidative stress injury of Caco2 cells by inhibiting caspase- 11 activation, the release of TNF- $\alpha$ and IL-3, and reducing intracellular free calcium ion.

The intestinal epithelium, often considered the primary defense for the host, forms a robust barrier to various infectious or noninfectious stimulations for maintaining intestinal mucosal homeostasis [21]. Oxidative stress and cytokines in the intestine in space and time orchestrate the initiation, development, exacerbation, and recurrence of the inflammatory process in various intestinal inflammatory diseases [22, 23]. ET (or lipopolysaccharides, LPS) is a component of the outer membrane of Gram-negative bacteria that live in the intestine, which could trigger oxidative stress and inflammatory response in a variety of cells [24-27]. In this study, we found that ET promoted caspase11 activation, inhibited cell growth, increased MDA and LDH expressions, and disrupted the mitochondrial morphology, which indicated that ET led to oxidative stress injury of Caco- 2 cells. In addition, the mitochondrial morphological injury of Caco- 2 cells became more serious with the increase of ET dose. The serum pharmacology method of TCM is a semi in vivo experimental method in 


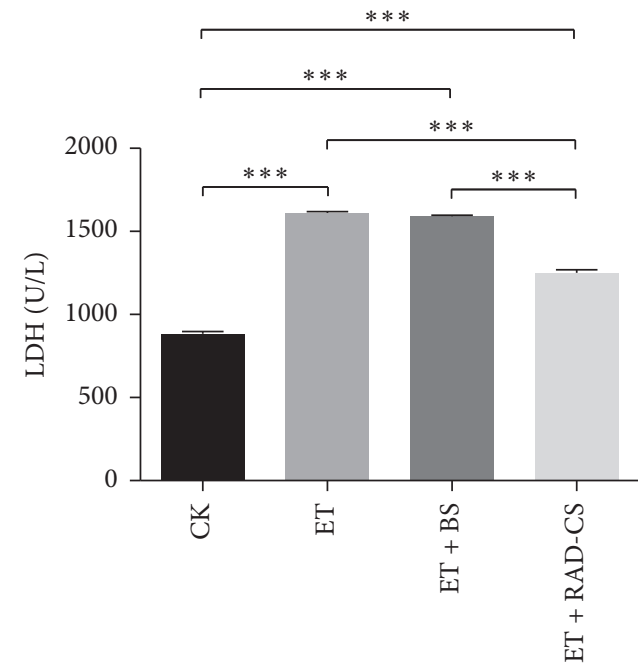

Figure 4: Effect of RAD drug-containing serum on lactate dehydrogenase (LDH) of Caco-2 cells induced by endotoxin in vitro. Values are mean $\pm \mathrm{SD}$, based on one-way ANOVA statistical analysis. ${ }^{* * *} P<0.001$.
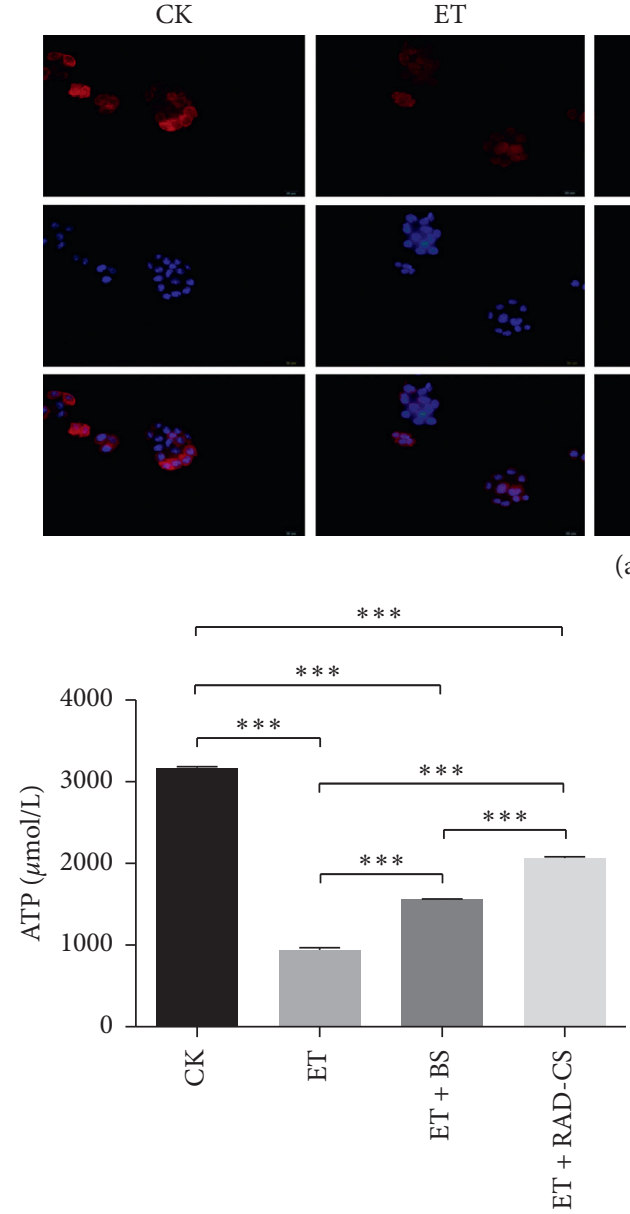

(b)

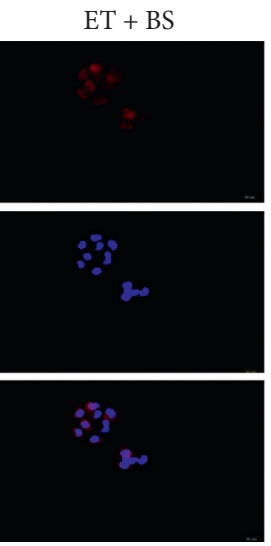

$\mathrm{ET}+\mathrm{RAD}-\mathrm{DS}$

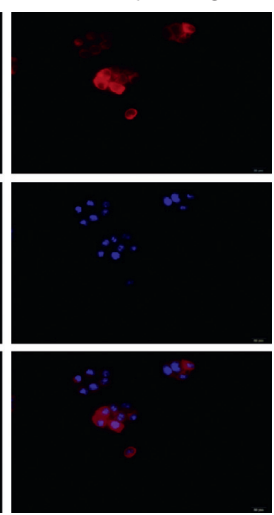

CK 18

(a)

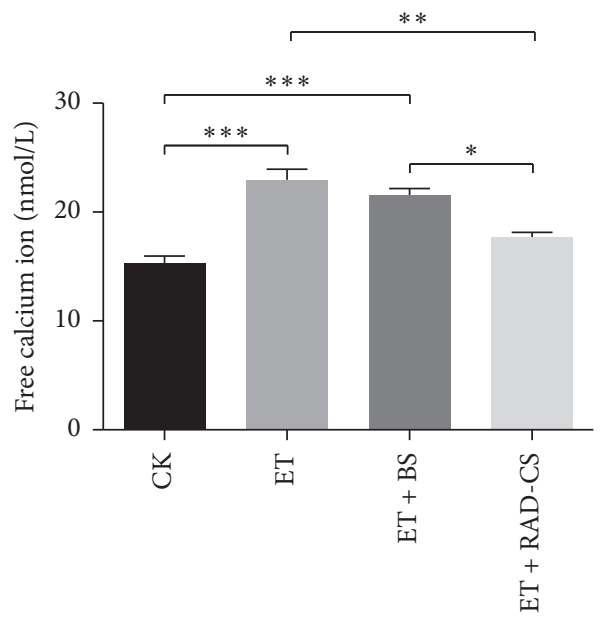

(c)

FIGURE 5: effects of RAD drug-containing serum on cytokeratin (CK)18, adenosine triphosphate (ATP), and intracellular free calcium ions in Caco-2 cells stimulated with endotoxin (ET). BS: blank serum. RAD-DS: RAD drug-containing serum. (a) Immunofluorescence staining of CK18 (red) and Hoechst staining of the nucleus (blue). (b) ATP level and (c) free calcium in groups. Values are mean \pm SD. Based on oneway ANOVA statistical analysis. ${ }^{*} P<0.05,{ }^{* *} P<0.01$, and ${ }^{* * *} P<0.001$. 
which an ancient Chinese medicine or a Chinese medicine compound is orally administered to an animal for a certain period of time, and then animal blood is collected, separating serum for preparing serum contained pharmaceutical ingredient, and in vitro experiments are performed. The serum pharmacology method provides a better way to study the effect of RAD on oxidative stress injury and inflammatory response of Caco- 2 cells induced by ET in vitro. In the current study, the rats were given RAD by gavage and then the RAD containing serum was prepared by blood collection from the abdominal aorta. We took this serum to treat Caco- 2 cells stimulated with ET, and then found that RAD containing serum significantly inhibited the caspase- 11 activation and the release of LDH. The dynamic regulation of free calcium ions was fundamental for cell life [28]. Previous studies have shown that stress can lead to an increase in intracellular free calcium levels $[29,30]$. In the present study, we found that RAD drugcontaining serum could reduce cytosolic free calcium ion ([Ca2+]i) level of Caco-2 cells after being stimulated with ET. The low level of $\mathrm{Ca}^{2+}$ made the $\mathrm{Ca}^{2+}$ develop an ability as a second messenger, through the energy-consuming pumping activity of $\mathrm{Ca}^{2+}$ ATPases. Excess free calcium ions in cells were toxic and caused the massive activation of proteases and phospholipases. Stressful events could induce alterations in the gut mucosa, barrier function, and small intestinal motility [31]. In addition, ET could significantly decrease the CK 18 expression and ATP activity, further disrupting the structure and energy supply of Caco2 cells, which were improved by RAD drug-containing serum. Taken together, the present study suggested that RAD drug-containing serum could significantly alleviate the ET-induced oxidative stress injury of Caco-2 cells.

To understand the underlying molecular mechanism of RAD In intestinal injury, the inflammatory reaction was analyzed by detecting the expression levels of TNF- $\alpha$ and IL3 . The intestine is a core organ where the inflammatory response occurs with the release of various inflammatory cytokines under stressful conditions, which has a potent impact on the small intestine. Especially, the inflammatory response may not only affect the intestine itself, but may also influence the function and integrity of remote organs and tissues, including the lung, liver, and brain, thus inducing SIRS and MODS. The intestine has been labeled the 'trigger' of the inflammatory responses under severe stress, which are mainly mediated by various inflammatory cytokines [3]. In the present study, the expressions of proinflammatory cytokine TNF- $\alpha$ and IL-3 were significantly increased in Caco2 cells stimulated with ET compared with unstimulated Caco- 2 cells. Moreover, ET also inhibited the cells growth and led to mitochondrial injury of Caco-2 cells. Previous studies indicated that the release of inflammatory cytokines induces a delay in small intestinal motility and intestinal obstruction [32-34]. Moreover, inflammatory cytokines exert cytotoxic effects that induce damage to microvilli, resulting in the destruction of tight intercellular junctions and increased intestinal barrier dysfunction [35, 36]. Many studies have found that traditional Chinese medicine could reduce intestinal inflammation $[37,38]$. Alabi QK reported that polyphenol-rich extract of Ocimum gratissimum leaves ameliorates colitis via attenuating colonic mucosa injury and regulating proinflammatory cytokines production and oxidative stress [39]. Our study suggested that RAD drugcontaining serum significantly decreased TNF- $\alpha$ and IL-3 levels of Caco- 2 cells after ET stimulation, while blank serum did not have the anti-inflammatory effect. Therefore, the intestinal inflammatory response may contribute to intestinal injury, and RAD could relieve intestinal injury by inhibiting the inflammatory response of Caco-2 cells.

\section{Conclusions}

In summary, we cultured Caco- 2 cells as experimental subjects and found that ET induced the oxidative stress injury and inflammatory response of Caco- 2 cells. In addition, we verified that RAD drug-containing serum alleviated ET-induced oxidative stress injury and inflammatory response of Caco- 2 cells by inhibiting the activation of caspase- 11 and the release of TNF- $\alpha$ and IL-3 and enhancing the antioxidant effect of cells. Although current findings are provoking, more in vivo studies are required to validate the protection effects of RAD on the intestine injury.

\section{Abbreviations \\ RAD: Rhubarb-aconite decoction \\ ET: $\quad$ Endotoxin \\ MDA: $\quad$ Lipid peroxide malondialdehyde \\ LDH: $\quad$ Lactate dehydrogenase \\ CK18: $\quad$ Cytokeratin 18 \\ SIRS: $\quad$ Systemic inflammatory response syndrome \\ MODS: $\quad$ Multiple organ dysfunction syndrome \\ TCM: $\quad$ Traditional Chinese medicine \\ MTT: Thiazolyl blue tetrazolium bromide \\ TEM: $\quad$ Transmission electron microscopy \\ SDS-PAGE: Sodium dodecyl sulfonate-polyacrylamide gel electrophoresis \\ PVDF: $\quad$ Polyvinylidene difluoride \\ TNF- $\alpha$ : Tumor necrosis factor- $\alpha$.}

\section{Data Availability}

The data used to support the findings of this study are included within the article.

\section{Conflicts of Interest}

The authors declare no conflicts of interest.

\section{Authors' Contributions}

Xiao-hong Du, Qing-jun Chen, Xin Kang, and Guo-hui Liu designed and performed the experiment. Xiao-hong Du, Xin Kang, and Guo-hui Liu analyzed the data. Xiao-hong Du, Xin Kang and Jian-bo Song wrote the paper. Yan Xie, Ru-ru Sun, Yan Zhi, and Guo-hui Liu reviewed the paper. All authors have read and agreed to the published version of the manuscript. Xiao-hong Du, Qing-jun Chen, and Jian-bo Song contributed equally to this work. 


\section{Acknowledgments}

This work was funded by the Natural Science Foundation of Jilin Science and Technology Development Plan (Bethune project, 20160101026JC), the grant of the National Natural Science Foundation of China (81202831), and the Liaoning Provincial Natural Science Foundation of China (2019-ZD0308).

\section{References}

[1] W. Druml, "Intestinaler crosstalk," Medizinische Klinik-Intensivmedizin und Notfallmedizin, vol. 113, no. 6, pp. 470-477, 2018.

[2] N. J. Klingensmith and C. M. Coopersmith, "The gut as the motor of multiple organ dysfunction in critical illness," Critical Care Clinics, vol. 32, no. 6, pp. 202-212, 2016.

[3] Y. Cheng, J. Zan, Y. Song et al., "Evaluation of intestinal injury, inflammatory response and oxidative stress following intracerebral hemorrhage in mice," International Journal of Molecular Medicine, vol. 42, no. 4, pp. 2120-2128, 2018.

[4] K. Timmermans, Ö. Sir, M. Kox et al., "Circulating iFABP levels as a marker of intestinal damage in trauma patients," Shock, vol. 43, no. 2, pp. 117-120, 2015.

[5] L. Xiaoling, A. M. Hammer, J. L. Rendon, and M. A. Choudhry, "Intestine immune homeostasis after alcohol and burn injury," Shock, vol. 43, no. 6, pp. 540-548, 2015.

[6] C.-H. Hang, J. X. Shi, J. S. Li, W. Wu, and H. X. Yin, “Alterations of intestinal mucosa structure and barrier function following traumatic brain injury in rats," World Journal of Gastroenterology, vol. 9, no. 12, pp. 2776-2781, 2003.

[7] X. Xu, Y. Zhu, and J. Chuai, "Changes in serum ghrelin and small intestinal motility in rats with ischemic stroke," The Anatomical Record: Advances in Integrative Anatomy and Evolutionary Biology, vol. 295, no. 2, pp. 307-312, 2012.

[8] C. J. Doig, L. R. Sutherland, J. Dean Sandham, G. H. Fick, M. Verhoef, and J. B. Meddings, "Increased intestinal permeability is associated with the development of multiple organ dysfunction syndrome in critically ill ICU patients," American Journal of Respiratory and Critical Care Medicine, vol. 158 , no. 2, pp. 444-451, 1998.

[9] S. Cao, C. Wang, J. Yan, X. Li, J. Wen, and C. Hu, "Curcumin ameliorates oxidative stress-induced intestinal barrier injury and mitochondrial damage by promoting Parkin dependent mitophagy through AMPK-TFEB signal pathway," Free Radical Biology and Medicine, vol. 147, no. 2, pp. 8-22, 2020.

[10] L. Kovacikova, M. Majekova, and M. Stefek, "Substituted pyridoindoles as biological antioxidants: drug design, chemical synthesis, and biological activity," Methods in Molecular Biology, vol. 1208, pp. 313-327, 2015.

[11] B. Jasiewicz, A. Sierakowska, W. Jankowski et al., "Antioxidant and cytotoxic activity of new di- and polyamine caffeine analogues," Free Radical Research, vol. 52, no. 6, pp. 724-736, 2018.

[12] M. M. Bordbar, B. Hemmateenejad, J. Tashkhourian, and S. F. Nami-Ana, "An optoelectronic tongue based on an array of gold and silver nanoparticles for analysis of natural, synthetic and biological antioxidants," Mikrobhim Acta, vol. 185, no. 10, p. 493, 2018.

[13] F. S. Liu, X. L. Fang, S. Y. Yuan et al., "Effects of Modified Dahuang Fuzi Decoction on intestinal motility and mucosal permeability in sepsis rats," JWRCM, vol. 27, pp. 1317-1320, 2018.
[14] L. Wu, L.. Xiao, H. Cai et al., "Effects of Dahuangfuzi Decoction on the STAT3 expression in pancreatic tissue of rats with severe acute pancreatitis," China Journal of Traditional Chinese Medicine and Pharmacy, vol. 27, no. 4, pp. 933-937, 2012.

[15] X. X. Liang, B. G. Zhang, and Q. F. Liu, "Study on modern pharmacodynamics and clinical application of rhubarb aconite decoction," Chinese Traditional Patent Medicine, vol. 11, pp. 1670-1673, 2008.

[16] X. G. Lu, L. B. Zhan, X. Kang et al., "Clinical research of Dahuang Fuzi decoction in auxiliary treatment of severe acute pancreatitis: a multi-center observation in 206 patients," Zhongguo wei zhong bing ji jiu yi xue, vol. 22, no. 12, pp. 723-728, 2010.

[17] X. H. Li and D. S. Zhang, "Clinical study of Dahuang-fuzi decoction on gastrointestinal dysfunction in sepsis patients," Hebei Journal of Traditional Chinese Medicine, vol. 39, no. 3, pp. 356-359, 2017.

[18] G. L. Yan, H. Sun, A. H. Zhang et al., "Progress of serum pharmacochemistry of traditional Chinese medicine and further development of its theory and method," Zhongguo Zhong Yao Za Zhi, vol. 40, no. 17, pp. 3406-3412, 2015.

[19] Y. K. Li and J. Y. Wu, "The general method scheme of blood collection time in serum pharmacological experiment," Chinese Pharmacological Bulletin, vol. 15, no. 2, pp. 569-570, 1999.

[20] Wu. Li, B. C. Cai, X. Liu et al., "Effects of serum containing Dahuang Fuzi Decoction on JAK2/STAT3 signal pathway in mice with severe acute pancreatitis," Chinese Traditional and Herbal Drug, vol. 44, no. 22, pp. 3195-3199, 2013.

[21] G. Chen, X. Ran, B. Li et al., "Sodium butyrate inhibits inflammation and maintains epithelium barrier integrity in a TNBS-induced inflammatory bowel disease mice model," Ebiomedicine, vol. 30, no. 4, pp. 317-325, 2018.

[22] M. Zhou, W. Xu, J. Wang et al., "Boosting mTOR-dependent autophagy via upstream TLR4-MyD88-MAPK signalling and downstream NF- $\kappa$ B pathway quenches intestinal inflammation and oxidative stress injury," EBioMedicine, vol. 35, no. 9, pp. 345-360, 2018.

[23] S. Das, J. S. Maras, M. S. Hussain et al., "Hyperoxidized albumin modulates neutrophils to induce oxidative stress and inflammation in severe alcoholic hepatitis," Hepatology, vol. 65, no. 2, pp. 631-646, 2017.

[24] M. Zhou, J. He, Y. Shen et al., "New frontiers in genetics, gut microbiota, and immunity: a Rosetta stone for the pathogenesis of inflammatory bowel disease," BioMed Research International, vol. 2017, no. 9, Article ID 8201672, 2017.

[25] Z. Dong and Y. Yuan, "Accelerated inflammation and oxidative stress induced by LPS in acute lung injury: inhibition by st1926," International Journal of Molecular Medicine, vol. 41, no. 6, pp. 3405-3421, 2018.

[26] Z. Wang, J. Xie, Y. Yang, F. Zhang et al., "Sulfated cyclocarya paliurus polysaccharides markedly attenuates inflammation and oxidative damage in lipopolysaccharide-treated macrophage cells and mice," Scientific Reports, vol. 7, no. 1, p. 40402, 2017.

[27] D. S. G. Nielsen, M. Fredborg, V. Andersen, and S. Purup, "Administration of protein kinase D1 induces a protective effect on lipopolysaccharide-induced intestinal inflammation in a co-culture model of intestinal epithelial caco-2 cells and raw 264.7 macrophage cells," International Journal of Inflammation, vol. 2017, no. 10, Article ID 9273640, 2017.

[28] W. Bochu, Z. Liancai, and C. Qi, "Primary study on the application of serum pharmacology in Chinese traditional 
medicine," Colloids and Surfaces B: Biointerfaces, vol. 43, no. 3-4, pp. 194-197, 2005.

[29] M. J. Berridge, M. D. Bootman, and H. L. Roderick, "Calcium signalling: dynamics, homeostasis and remodelling," Nature Reviews Molecular Cell Biology, vol. 4, no. 7, pp. 517-529, 2003.

[30] J. Deweirdt, J. F. Quignard, B. Crobeddu et al., "Involvement of oxidative stress and calcium signaling in airborne particulate matter - induced damages in human pulmonary artery endothelial cells," Toxicology in Vitro, vol. 45, no. 3, pp. 340-350, 2017.

[31] K. Rtibi, S. Selmi, D. Grami, H. Sebai, M. Amri, and L. Marzouki, "Irinotecan chemotherapy-induced intestinal oxidative stress: underlying causes of disturbed mucosal water and electrolyte transport," Pathophysiology, vol. 24, no. 4, pp. 275-279, 2017.

[32] J. C. Kalff, W. H. Schraut, T. R. Billiar, R. L. Simmons, and A. J. Bauer, "Role of inducible nitric oxide synthase in postoperative intestinal smooth muscle dysfunction in rodents," Gastroenterology, vol. 118, no. 2, pp. 316-327, 2000.

[33] S. Wehner, T. O. Vilz, B. Stoffels, and J. C. Kalff, "Immune mediators of postoperative ileus," Langenbeck's Archives of Surgery, vol. 397, no. 3, pp. 591-601, 2012.

[34] S. Wehner, N. T. Schwarz, R. Hundsdoerfer et al., "Induction of IL-6 within the rodent intestinal muscularis after intestinal surgical stress," Surgery, vol. 137, no. 4, pp. 436-446, 2005.

[35] A. M. Marchiando, L. Shen, W. V. Graham et al., "Caveolin-1dependent occludin endocytosis is required for tnf-induced tight junction regulation in vivo," Journal of Cell Biology, vol. 189, no. 1, pp. 111-126, 2010.

[36] J. R. Turner, "'Putting the squeeze' on the tight junction: understanding cytoskeletal regulation," Seminars in Cell \& Developmental Biology, vol. 11, no. 4, pp. 301-308, 2000.

[37] M. Zhang, H. Fan, S. Tan et al., "The Chinese medicinal herb decoction QRZSLXF enhances anti-inflammatory effect in TNBS-induced colitis via balancing Th17/Tregs differentiation," Journal of Ethnopharmacology, vol. 251, no. 1, Article ID 112549, 2020.

[38] Z. Jiang, Q. Chen, J. Zhang et al., "Wuda granule, a traditional Chinese herbal medicine, ameliorates postoperative ileus by anti-inflammatory action," Pathology-Research and Practice, vol. 216, no. 3, Article ID 152605, 2020.

[39] Q. K. Alabi, R. O. Akomolafe, J. G. Omole et al., "Polyphenolrich extract of Ocimum gratissimum leaves ameliorates colitis via attenuating colonic mucosa injury and regulating proinflammatory cytokines production and oxidative stress," Biomedicine \& Pharmacotherapy, vol. 103, no. 7, pp. 812-822, 2018. 